\title{
Políticas de acceso de la población vulnerable a la educación superior, una visión desde la experiencia de la Universidad del Magdalena
}

\author{
Access policies of vulnerable population to higher education, a vision from the experience of \\ Magdalena's University
}

\begin{abstract}
Resumen: En este documento se analizan las políticas implementadas por la Universidad del Magdalena para garantizar el acceso de los grupos vulnerables a la educación superior, definiendo como vulnerables a los bachilleres desplazados, las comunidades indígenas, los estudiantes afrocolombianos, la mujer cabeza de familia de estrato bajo, y a los bachilleres provenientes de colegios públicos. Teóricamente, el estudio está asociado a los aportes de la affirmative action, la cual contempla la intención deliberada de eliminar cualquier ápice de discriminación en el desarrollo de las sociedades y las instituciones. Durante el estudio, se encontró que más del $70 \%$ de este grupo de estudiantes, convive en un núcleo familiar con ingresos inferiores a los seiscientos mil pesos/mes. Por lo tanto, las principales estrategias de la Universidad, están enfocadas en allanar las limitaciones económicas de la población vulnerable, mediante las exoneraciones y subsidios acompañadas de un marco normativo que facilita el acceso de esta comunidad a la Universidad. Por otra parte, metodológicamente es un tipo de artículo derivado de una investigación.
\end{abstract}

Palabras clave: affirmative action, equidad, población vulnerable, educación superior.

JEL: I21

\begin{abstract}
This document analyzes the politics implemented by university of Magdalena to guarantee the access of higher education to vulnerable groups. Vulnerable is defined as the displaced graduate, the indigenous communities, the afro-Colombian students, the female head of a household of a low stratum and the graduates who come from public schools. In theory, the study is associated with the contribution of the affirmative action, which includes the deliberate intention to eliminate any discrimination in the development of societies and institutions. During the study, it was found that more than $70 \%$ of this students group lives in a family nucleus with low incomes around six hundred pesos per month. Therefore, the main strategies of the university are focused to overcome the economic limitations of this vulnerable population, by exemptions and subsidies accompanied by a policy framework that facilitates the Access to the community of the university. Methodologically so it is a type of derivative research article.
\end{abstract}

Keywords: affirmative action, equity, vulnerable population, higher education.

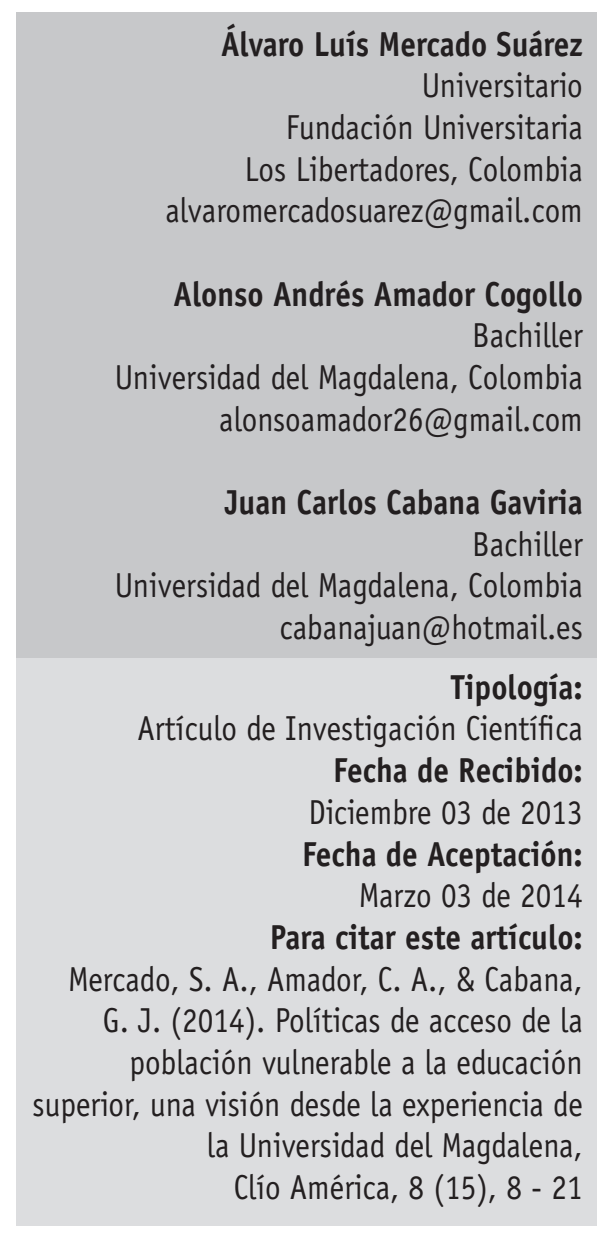




\section{Introducción}

Existe consenso entre las autoridades educativas colombianas sobre la importancia que tiene la educación para el desarrollo del país, y es por esta razón que ha aumentado el interés de las Instituciones de Educación Superior (IES), en asegurar el acceso del mayor número de personas que se disponen a iniciar sus estudios universitarios.

Sin embargo, el deseo de estudiar una carrera profesional no está al margen de enfrentar obstáculos que, en muchas ocasiones, impiden el ingreso a una IES. Por un lado, factores de tipo social relacionados a una condición étnica, de raza, o de víctimas del conflicto colombiano han sido desde antaño motivos por los cuales una parte de la población ha permanecido aislada del dinamismo académico profesional. Por otra parte, el factor económico también ha sido causante de que una parte de los jóvenes colombianos deserten de sus estudios superiores, 0 , en el peor de los casos, no puedan acceder a una IES. No obstante, la política educativa del siglo XXI ha evolucionado al ritmo de los nuevos desafíos que se imponen en el proceso de la formación profesional de los colombianos, atendiendo las falencias del sistema educativo.

En tal sentido, se puede observar claramente en los planes de desarrollo de las IES (como lo son el plan de desarrollo de la Universidad de Antioquia y de la Universidad UIS), que las directrices educativas impartidas por el gobierno nacional, demandan el desarrollo de estrategias que garanticen el acceso a la educación superior de aquella parte de la población que, por motivos de condición social 0 económicos, han permanecido históricamente por fuera del sistema educativo profesional.

Por otro lado, Derechos Humanos claramente exponen y reglamentan las condiciones y oportunidades que deben tener las poblaciones vulnerables en un sentido de darle igualdad a todos los seres humanos sin distingo de razas, religión y clase social. Tal cual lo expone las Declaración de Derechos Humanos en sus artículos $1^{\circ}$ y $2^{\circ}$, donde además en su artículo $26^{\circ}$ vela y promulga por una educación de nivel profesional generalizada y con las garantías de oportunidades para las poblaciones vulnerables.
$Y$ en el entendido de que la transformación y el desarrollo de la sociedad a través de la educación es la razón de ser de la universidad, las estrategias que buscan garantizar la equidad en el acceso a la educación superior, se convierten en un mecanismo para elevar los niveles de calidad de vida de toda una nación.

Bajo este contexto, cabe anotar que los resultados obtenidos por la política nacional de educación superior, son alentadores. De acuerdo al Ministerio de Educación Nacional (MEN, 2013), la cobertura nacional de educación superior pasó del 37\% al 42\% entre el 2010 y el 2012, al tiempo en que la proporción de estudiantes con apoyo financiero estatal se incrementó, pasando del $66 \%$ al $73 \%$ en el mismo período.

Lo anterior guarda relación con el hecho de que, en el 2012 , el $57 \%$ de los estudiantes de las IES residen en los estratos 1-2, y que el $58 \%$ procediera de familias con ingresos inferiores a los dos salarios mínimos vigentes. Es decir, hay una gran participación de los estudiantes categorizados en situación de vulnerabilidad por sus condiciones económicas. Entre tanto, esta perspectiva de la educación también ha influenciado notoriamente los programas tendientes a facilitar el acceso equitativo a la Universidad del Magdalena, materializándose en el principio de equidad del Plan de Desarrollo 2012 - 2016, en el cual se compromete la Universidad a garantizar la "Igualdad de condiciones y oportunidades para el acceso a la universidad, sus bienes y servicios, teniendo en cuenta las particularidades de los grupos vulnerables".

A este postulado responden los distintos acuerdos normativos aprobados por el Consejo Superior para mejorar los estándares de equidad en la Universidad, estipulando parámetros mínimos de acceso a las poblaciones afro, indígena, desplazada, madres cabeza de familia y a los bachilleres provenientes de familias con escasos recursos financieros. Además, las exoneraciones en matrícula por cupos especiales, redistribución, convenios especiales, bienestar universitario, créditos académicos y por estrato socioeconómico constituyen un apoyo dirigido a las dificultades económicas. 
Políticas de acceso de la población vulnerable a la educación superior, una visión desde la experiencia de la Universidad del Magdalena

Este documento se deriva de la investigación titulada Análisis de los programas de equidad en el acceso y la permanencia de los estudiantes de población vulnerable en los programas de pregrado de la Universidad del Magdalena durante los años comprendidos entre 2005 y 2011-I. Está conformado por cuatro secciones, iniciando con la presente introducción. A continuación se desarrollan los acápites conceptuales, reglamentarios y teóricos que soportan el estudio. En la sección de resultados, se muestran algunos datos estadísticos relevantes de la Universidad del Magdalena, analizando su contenido. Finalmente, se describen las conclusiones del estudio.

Durante la realización del estudio se presentan algunos conceptos que, aunque parecen no requerir mayor profundización en su significado, es relevante definirlos con el fin de delimitar su utilización a lo largo del documento.

Por ejemplo, referirnos a la población vulnerable abarcaría gran parte de la población que, por alguna característica específica, se encuentra en una condición desfavorable respecto a otro sector de la sociedad. Pero este alcance representaría una definición más allá de lo que realmente se referencia en el presente estudio. Dicho lo anterior, conviene definir qué se entiende por población vulnerable y los términos que de ésta se derivan.

Población vulnerable: definida por el MEN (2005) como las comunidades étnicas, los jóvenes y adultos iletrados, los menores con necesidades educativas especiales, los afectados por la violencia, los menores en riesgo social, los habitantes de frontera y la población rural dispersa. Sin embargo, por adaptación al objetivo del estudio se define como población vulnerable a los bachilleres que provienen de familias con ingresos menores a los 2 SMLV y que vivan en estratos 1 y 2, a las comunidades afrocolombianas, a la población desplazada por la violencia, a las comunidades indígenas y las madres cabeza de hogar de estratos bajos.

Comunidades afrocolombianas: conjunto de familias con descendencia africana. Poseen su propia cultura, historia, tradiciones y costumbres dentro de la relación campo-poblado, conservan una iden- tidad que la distinguen de otros grupos. (Pastoral Afrocolombiana). Entre las principales normas que regulan el tratamiento a esta población, encontramos la ley 70 de 1993, el Decreto 804 de 1995, el Decreto 1122 de 1998 y la Directiva Ministerial 011 de 2004 (MEN).

Población desplazada por la violencia: La Ley 387 de 1997 define como desplazada a toda persona que se ha visto forzada a migrar dentro del territorio nacional, a abandonar la localidad de residencia y las actividades económicas habituales porque su vida, integridad física, seguridad o libertad personal han sido vulneradas o se encuentran directamente amenazadas.

Madre cabeza de familia: quien siendo soltera 0 casada, ejerce la jefatura del hogar y tiene bajo su cargo, en forma permanente, hijos menores propios u otras personas incapaces o incapacitadas para trabajar, ya sea por ausencia permanente 0 incapacidad física, sensorial, síquica o moral del cónyuge o compañero permanente; y deficiencia sustancial de ayuda de los demás miembros del núcleo familiar. (Ley 82 de 1993).

Acción afirmativa (affirmative action ${ }^{1}$ ): Gómez y Celis (2009) la definen citando que es "toda medida de carácter temporal con la que se busca asegurar la igualdad de oportunidades, a través de un trato preferencial, a los miembros de un grupo que ha experimentado situación de discriminación y marginalidad". (León \& Holguín, 2004, p. 57)

El nacimiento de las acciones afirmativas data de mediados del siglo XX en la India, exactamente después de su independencia, con las cuales se buscaba eliminar la discriminación social basada en la casta. Tales fueron los beneficios sociales derivados de esta estrategia que, posteriormente, en Estados Unidos, Sudáfrica y Brasil también fueron llevadas a cabo para acabar con la exclusión a la comunidad afrodescendiente. (Reiter \& Lezama, 2013)

1. La affirmative action no es un término exclusivo al campo educativo, en otras áreas del conocimiento y la política también es posible referirse a las acciones afirmativas. Por ejemplo, una visión desde el enfoque de las leyes se encuentra en Weisner, L. (2007). La acción afirmativa en la Constituyente de 1991. Universidad Sergio Arboleda. 
Partiendo de este concepto, Gómez y Celis (2009) establecen nueve modalidades de acciones afirmativas que las IES implementan para el desarrollo de sus políticas de acceso a la población vulnerable: las admisiones especiales, los exámenes especializados, las becas y subsidios, los subsidios familiares, el acompañamiento académico, la conexión IES-secundaria, el ofrecimiento laboral a estudiantes, el monitoreo de los egresados y la flexibilidad curricular.

Por un lado, con las admisiones especiales se estipula un cupo mínimo de ingreso de una comunidad específica, estableciendo requisitos y evaluaciones diferenciados para esta población; mientras que con los exámenes especializados, se pueden identificar los distintos grupos sociales que acceden a una IES, de tal manera que desde el ingreso del estudiante, se focalizan los programas diseñados para cada tipo de población.

También se encuentran las becas y los subsidios otorgados para aminorar los costos de matrícula o de mantenimiento respectivamente, estableciendo parámetros asequibles para determinada población, con el fin de facilitar la obtención de los beneficios.

En la Universidad del Magdalena, esta modalidad se presenta mediante los subsidios de alimentación y refrigerios para los estudiantes categorizados como de bajos recursos económicos. También se encuentran las exoneraciones en el costo de la matrícula meritorias por pertenecer a una de las comunidades vulnerables. (Ver Tabal 1)

Tabla 1.

\section{Acuerdos superiores que reglamentan exoneraciones a la población vulnerable de la Universidad del Magdalena}

\begin{tabular}{|l|c|c|c|}
\hline \multicolumn{1}{|c|}{ Población beneficiada } & Acuerdo Superior No & Exonerados por cohorte & Porcentaje \\
\hline Desplazado & $008,021 / 2003$ & 1 & $90 \%$ \\
\hline Indígena & $024 / 2001,008 / 2003$ & 1 & $90 \%$ \\
\hline Afrocolombiano & $024 / 2001,008 / 2003$ & 1 & $90 \%$ \\
\hline Madre cabeza de familia. & $026 / 2001,008 / 2003$ & 1 & $90 \%$ \\
\hline Bachiller estratos 1. & $035 / 2003$ & 7 & $90 \%$ \\
\hline Bachiller estrato 2. & $035 / 2003$ & 7 & $50 \%$ \\
\hline $\begin{array}{l}\text { Bachiller colegio público rural } \\
\text { Santa Marta, estratos 1,2, ó } 3\end{array}$ & $035 / 2003$ & 2 & $90 \%$ \\
\hline mejor examen de admisión. & & & \\
\hline
\end{tabular}

Fuente: Acuerdos expedidos por el Consejo Superior de la Universidad del Magdalena del 2001 - 2003. 
Políticas de acceso de la población vulnerable a la educación superior, una visión desde la experiencia de la Universidad del Magdalena

No obstante, la política de exoneraciones en la Universidad abarca un segmento de la comunidad estudiantil más amplio; otorga exoneraciones determinadas por la ubicación geográfica dentro de Colombia por rendimiento deportivo, por excelencia académica, entre otras categorías.

Otro tipo de programa especializado de las acciones afirmativas, es el financiamiento indirecto, mediante subsidios familiares, del gasto en que incurren las familias por el servicio en educación. Desde la perspectiva académica también se ejecutan diversos proyectos institucionales tendientes a garantizar tanto el ingreso a una IES, como el mantenimiento en ésta.

Por ejemplo, los programas diseñados para establecer un vínculo entre las IES y las escuelas secundarias, facilitan la transición de un nivel educativo básico a otro más avanzado que, en muchos casos, impide el ingreso de una parte de los bachilleres a la IES debido al rezago académico de la educación impartida en muchas escuelas.

Entre tanto, el acompañamiento académico dirigido evita que, una vez el estudiante ha ingresado a la IES, deserte por causa del bajo rendimiento académico.

Siguiendo este enfoque, además, se encuentran los planes de seguimiento a los egresados, elaborados con el fin de monitorear la inserción de los recién graduados en el mercado laboral, creando una conexión empresas-egresados que disminuye las barreras que enfrentan los nuevos profesionales al intentar posicionarse en el trabajo.

Finalmente, las IES también desarrollan programas de oferta laboral dirigidos a estudiantes en riesgo de deserción por motivos económicos, generalmente para realizar labores en el área administrativa o de monitorias académicas ${ }^{2}$, para el caso de aquellos estudiantes que mantienen un promedio de calificación excelente y pueden ejercer funciones como monitores.

2. El cargo que pueda desempeñar un estudiante en una IES varía de una institución a otra, y depende de las normas internas que regulen tal iniciativa laboral estudiantil. En algunas instituciones, los estudiantes desarrollan labores en las áreas de biblioteca o de algún departamento especializado al interior de la institución, también pueden vincularse a través de tareas investigativas.
Además, la flexibilidad curricular se convierte en una forma de acción afirmativa por cuanto permite que el estudiante, al tiempo que avanza en sus estudios superiores, devengue un salario que le ayude a financiar sus gastos en educación, bien sea a través de la IES o por un empleador externo.

Otro complemento a las políticas de acciones afirmativas en la educación superior colombiana, lo hallamos en el trabajo de Sánchez, Quirós, Reverón y Rodríguez (2002), quienes incluyen los programas institucionales como los cupos especiales, los cursos preuniversitarios y la eliminación de estándares mínimos en los puntajes de admisión.

En el mismo estudio encontramos tipificados distintos programas de acción afirmativa dirigidos a la población estudiantil vulnerable. En la Tabla 2, se puede observar que las comunidades indígenas, los reinsertados, las personas en situación de discapacidad, los desplazados, los estudiantes en precarias condiciones socioeconómicas y las madres cabeza de familia son la población objetivo de dichos programas.

Aunque entre las principales conclusiones del trabajo se resaltan los efectos positivos que tienen los programas de acción afirmativa sobre la permanencia de los estudiantes, también se advierte que "...la mayoría de los mismos son poco integrales, ya que están más orientados a solucionar el pago de la matrícula [...] que a garantizar el sostenimiento de los estudiantes en sus diversas dimensiones...". (Sánchez, F., Quirós, M., Reverón, C., y Rodríguez, A., 2002, p. 24)

\section{Metodología}

La evidencia empírica que respalda la ejecución de programas diseñados por las IES y orientados a beneficiar a la población vulnerable, se deriva de distintas investigaciones académicas que ofrecen aportes para mejorar los estándares de acceso a la educación superior.

Los enfoques de cada tesis varían según el propósito de cada institución o grupo de investigación, así como también dependen de las diferencias regiona- 
les 0 de países; se pueden encontrar investigaciones afianzadas en el acceso a la educación superior, otros focalizados en la permanencia, algunos dirigidos a la deserción y retención estudiantil, u otros que combinan más de un objetivo.

-ITabla 2.

\section{Tipos y subtipos de programas de acción afirmativa en el acceso a universidades públicas en Colombia}

\begin{tabular}{l|l} 
& Estudiantes peores condiciones socioeconómicas \\
& Indígenas \\
& Reliquidación \\
\hline Subsidios en el valor de matricula & Desplazados \\
\hline & Bachilleres de municipios pobres \\
\hline Madres cabeza de familia \\
\hline Reinsertados \\
\hline Discapacitados \\
\hline Subsidio en sostenimiento & Subsidio alimentación/restaurante \\
\hline Residencias \\
\hline Apoyo en textos escolares \\
\hline Apoyo económico a estudiantes de bajos ingresos - préstamo \\
condonable
\end{tabular}

Fuente: modificado por los autores. Tomado de Sánchez et al. (2002) p.23.

Dicho lo anterior, la $0 \mathrm{CDE}^{3}$ (2012) analizó las dificultades para ingresar a la educación superior teniendo en cuenta la preparación académica de los egresados, y encontró que

... muchos estudiantes que llegan a instituciones de educación superior, especialmente a las universidades, carecen de una buena preparación para la universidad. Por tanto, existe una gran diferencia entre los conocimientos y las competencias que han adquirido en el colegio y los conocimientos y competencias que necesitan para cursar efectivamente estudios de educación

3. Organización para la Cooperación y el Desarrollo Económicos. superior. Cuanto mayor es esta diferencia para un estudiante en particular, mayor es el riesgo de que, si consigue acceder a la educación superior, no consiga estar a la altura de las exigencias de su programa y abandone los estudios. (OCDE, 2012, p. 102)

Por su parte, Sánchez et al. (2002) encontraron que las universidades que implementan un examen específico de admisión y realizan entrevistas, reducen las probabilidades de ingreso de los bachilleres con calificación media y baja en el ICFES ${ }^{4}$, en compara-

4. El ICFES fue el examen nacional que medía las competencias académicas de los bachilleres colombianos, actualmente se denomina Saber Pro 11. 
Políticas de acceso de la población vulnerable a la educación superior, una visión desde la experiencia de la Universidad del Magdalena

ción con las probabilidades de acceso en aquellas instituciones cuyo requisito de ingreso era, únicamente, el puntaje ICFES.

Además, se puede afirmar que la implementación de programas de acción afirmativa produce efectos positivos sobre la permanencia estudiantil en la educación superior, pues por cada programa ejecutado por una IES, la probabilidad de permanencia de sus estudiantes incrementa en porcentajes cercanos al $8 \%$. (Sánchez et al., 2002)

Reiter y Lezama referencian el estudio de Marques da Silva y Dias da Silva (2009) ${ }^{5}$ para exponer brevemente el impacto de dichos programas en las universidades de Brasil. En dicho estudio, encontraron que en el período 2005-2009 cerca de 247,000 estudiantes afrodescendientes accedieron a la universidad como parte del programa federal 'Universidad para todos', alcanzando los 350,000 en el 2011.

Además, citan los resultados de Roberto de Mattos $(2003)^{6}$ y afirman que a partir del año 2003 la Universidad del Estado de Bahía (Brasil) estableció que el $40 \%$ de los cupos ofrecidos se reservara a bachilleres afrodescendientes, lo cual permitió el acceso de más de 28,000 estudiantes de esta comunidad que, en su mayoría, provenían de colegios públicos y de familias con bajos ingresos económicos.

\section{Resultados}

A continuación se presenta un análisis estadístico de las principales políticas de equidad de la Universidad del Magdalena, basado en las fuentes de información del Grupo de Admisiones, Registro y Control Académico, y en estudios desarrollados por distintas dependencias de la Universidad en el marco de las estrategias dirigidas a la población vulnerable.

Como se ha mencionado a lo largo del texto, las exoneraciones sobre el valor de la matrícula otorgada a estudiantes que clasifican para cierta categoría,

5. Instituto de Pesquisa Económica Aplicada, (2009). Igualdade racial. In Políticas Sociais, Acompanhamento e Análise, 19.

6. Instituto Nacional de Estudos e Pesquisas Educacionais Anísio Teixeira, (2003). Ações Afirmativas na Universidade do Estado da Bahia: Razões e Desafios de uma Experiência Pionero. ocupan un rubro muy importante en el gasto institucional para beneficiar a una comunidad estudiantil específica.

Alrededor del $80 \%$ de las exoneraciones que otorga la Universidad, se entregan por bienestar universitario, por nivel de estrato socioeconómico, por cantidad de créditos académicos matriculados, y por promedio académico. (Ver Gráfico 1)

Aunque es importante la participación de las exoneraciones por estrato socioeconómico y por créditos académicos, hay que mencionar que la mayor proporción de exoneraciones se ofrece por bienestar universitario, es decir, por factores asociados al rendimiento deportivo, a las actividades artísticas, a las monitorias académicas y administrativas, entre otros factores.

Entre tanto, las exoneraciones entregadas por cupos especiales representan cerca del $11 \%$, que equivale a 396 estudiantes del total de categorías definidas para el otorgamiento de este beneficio. Que van desde Bachiller desplazado; comunidades indígenas con asentamiento Magdalena; bachiller afrocolombiano; mujer bachiller cabeza de familia de estrato 1, 2 ó 3; bachiller deportista; deportista galardonado nacional 0 internacionalmente; bachiller artista y bachiller municipio del Magdalena menor a 50.000 habitantes.

Un segundo grupo de cupos especiales corresponden a exoneraciones por Redistribución según las zonas de procedencia de los aspirantes de las distintas subregiones del departamento del Magdalena y los convenios especiales. Entre ellas se encuentran: Redistribución Zona Centro, Redistribución Zona Norte, Redistribución Zona Río, Redistribución Zona Sur, Redistribución Zona Santa Marta.

Entre las exoneraciones por Convenios Especiales, se encuentran la Beca Taganga a estudiantes de cohortes anteriores a 2010-II y que no se han readmitido, Beca Taganga - cohortes 2010-II y los estudiantes de cohortes anteriores que se readmitan a partir del período 2010-II). 4. También existen Exoneraciones por Promedios (Matrícula de honor, Primer puesto, segundo puesto, tercer puesto, cuarto puesto, quinto puesto por rendimiento académico). 
En materia de Exoneraciones por Bienestar Universitario y otros se ofrecen a Deportista de alto rendimiento, Artista de alto rendimiento, Monitor académico, Monitor administrativo, Descuento por hermanos. Adicionalmente se contemplan Exoneraciones por Créditos Académicos y Similares (Estudiante que cursará entre el $60 \%$ y menos del $80 \%$ de los créditos del semestre, Estudiante que cursará entre el $40 \%$ y menos del $60 \%$ de los créditos del semestre, Estudiante que cursará menos del $40 \%$ de los créditos del semestre, Estudiante de grado (Antiguos), Estudiante de grado (Reforma), Estudiante de prácticas profesionales.

\section{- Figura 1.}

\section{Porcentaje según categoría de exoneración de matrícula de la Universidad del Magdalena}

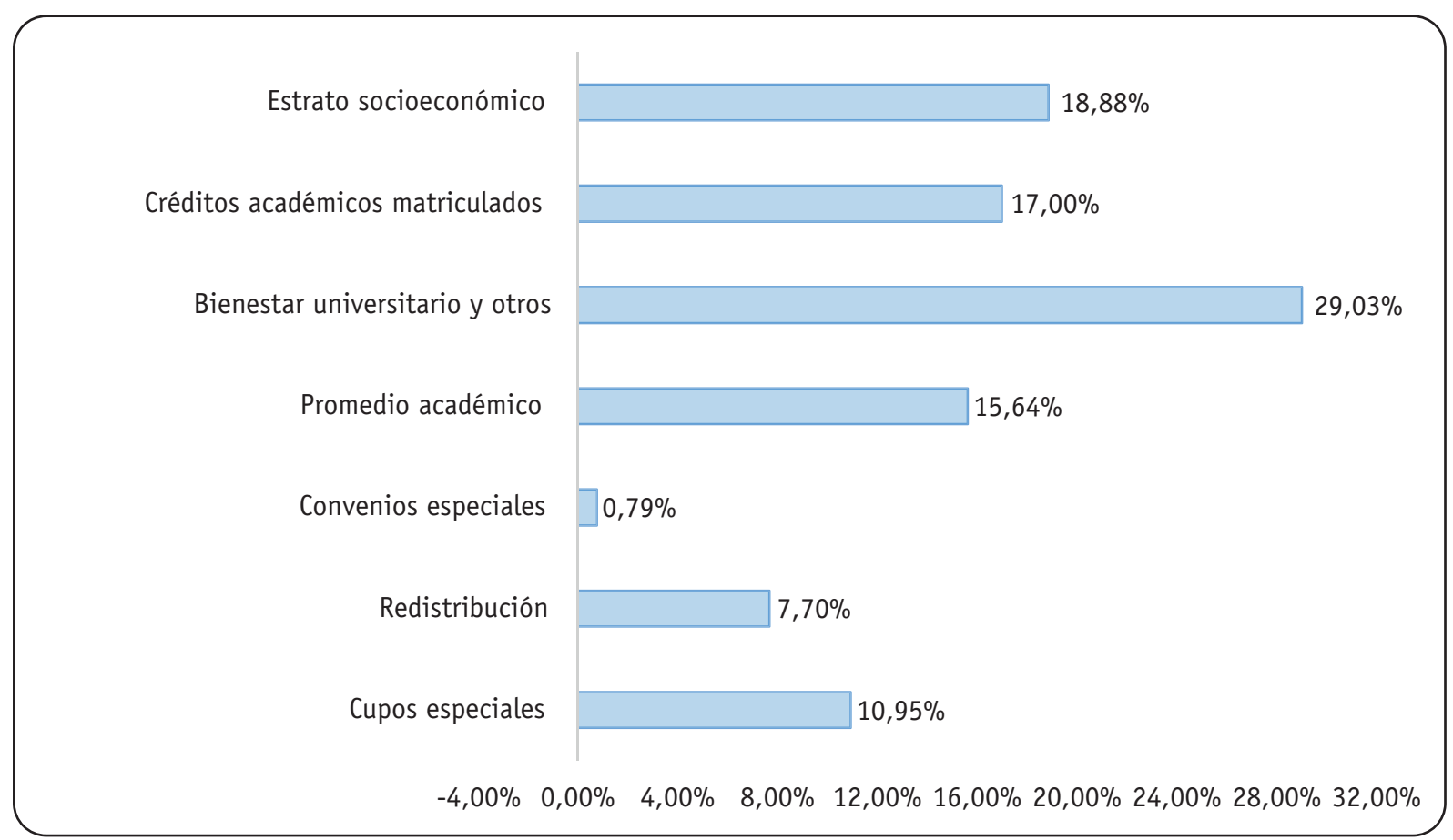

Fuente: Figura de los autores con base cifras de Marenco, O. (2013)

Finalmente, se implementan también Exoneración por Estrato Socioeconómico (Bachiller estrato uno colegio público con sede en la ciudad de Santa Marta, Bachiller estrato dos colegio público con sede en la ciudad de Santa Marta).

Otro aspecto muy importante es que existe una gran concentración en el ofrecimiento de las exoneraciones. Entre las facultades de Ingeniería y de Ciencias Empresariales y Económicas, abarcan las dos terceras partes del total de exoneraciones entregadas por la
Universidad, esto debido a que estas dos unidades académicas concentran la mayor participación de la población estudiantil de la institución.

Tal y como se muestra en el Gráfico 2, en el período 2011-I la Facultad de Ingeniería concentró el 33\% del total de estudiantes matriculados, mientras que Ciencias Empresariales recaudó el $28 \%$ del estudiantado, seguida por Ciencias de la Salud con $20 \%$, principalmente los programas de Medicina y Psicología. 


\section{JFigura 2.}

\section{Porcentaje de estudiantes matriculados en pregrado de la Universidad del Magdalena según facultad, 2011-I}

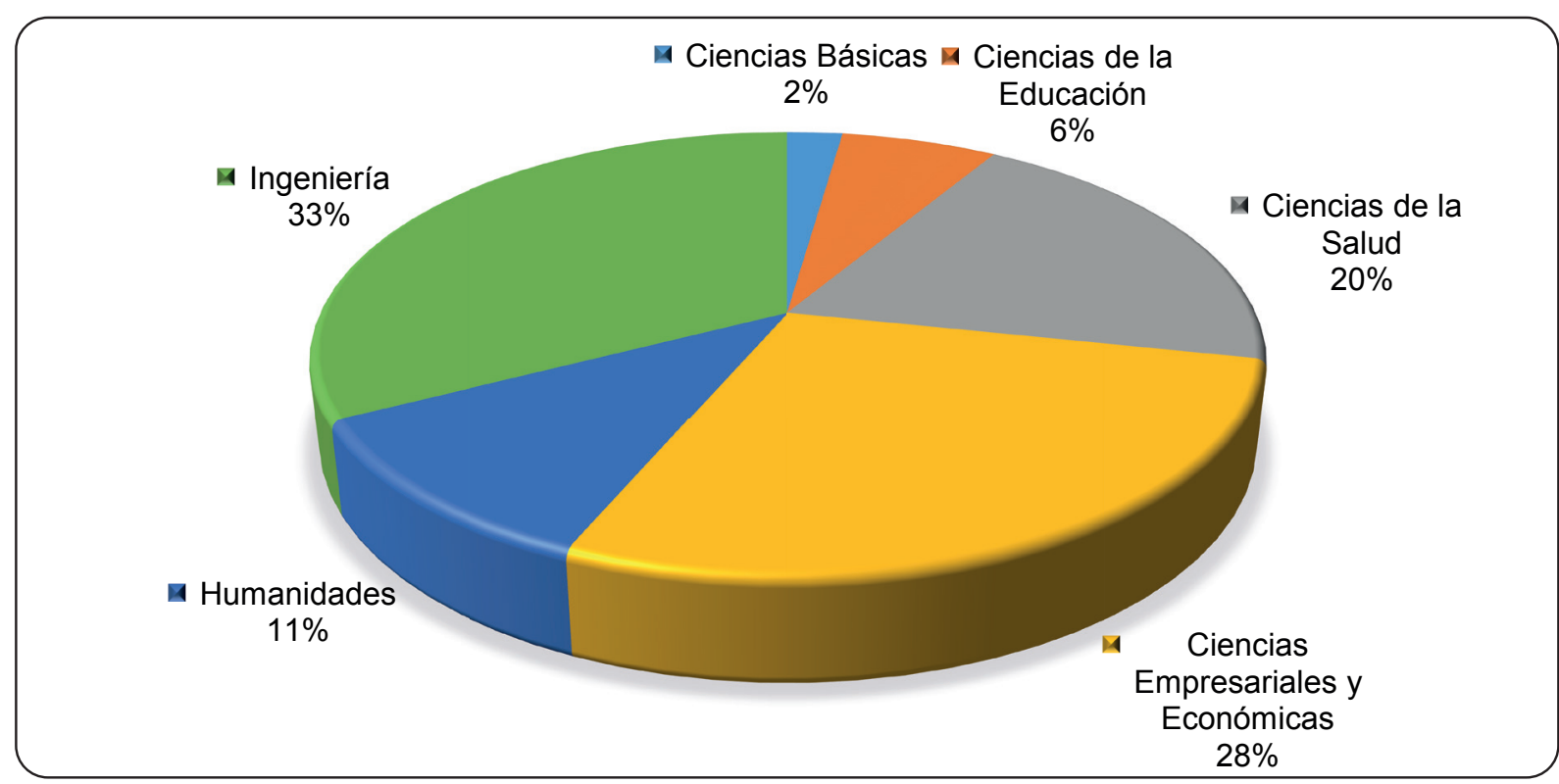

Fuente: elaboración de los autores con base en el Boletín Estadístico 2011 del grupo Ayre de la Universidad del Magdalena.

Siguiendo esta línea de análisis, se puede apreciar en la Figura 3 que los programas académicos con mayor participación son, en su orden, Administración de Empresas, Contaduría y Negocios Internacionales; estos tres programas académicos reciben aproximadamente el $25 \%$ del total de las exoneraciones de un total de 34287 para el periodo comprendido entre el 2005 - 2011, seguidas por Ingeniería de Sistemas e Ingeniería Industrial con $7,37 \%$ y $7,22 \%$ respectivamente también de un total de 34287 exonerados.

Cabe anotar que la alta participación de estos programas está relacionada con el número de estudiantes matriculados en estas carreras, pues el $82 \%$ de los estudiantes se encuentra en las facultades de Empresariales, Salud e Ingeniería.

Otro análisis también se puede realizar desde la perspectiva del gasto económico en que incurre la
Universidad para desarrollar este tipo de políticas. De acuerdo con el Grupo de Admisiones, Registro y Control Académico, entre el año 2005 y el primer período académico del 2011, la Universidad destinó más de $\$ 22,000$ millones de pesos al ofrecimiento de exoneraciones teniendo como parámetro de otorgamiento algún tipo de característica sociocultural, a excepción de las entregadas por excelencia académica.

La Figura 4 muestra que el $66 \%$ del gasto mencionado se destinó a financiar el esquema de política de equidad de la Universidad del Magdalena, lo que demuestra una focalización del gasto hacia el beneficio de la población vulnerable de la institución. No obstante, teniendo en cuenta la definición citada sobre las acciones afirmativas, se puede decir que, si se incluyen los recursos invertidos en las monitorias, dicha proporción alcanza el 73\%. 


\section{- Figura 3.}

\section{Participación en las exoneraciones de matrícula de la Universidad del Magdalena según carrera profesional}

Tec. en Amdón. Hotelera y Turística Psicología Odontología Negocios Internacionales Medicina Lic. Preescolar Lic. Ciencias Básicas Ing. Pesquera Ing. Industrial Ing. Electrónica Ing. Sistemas Ing. Civil Ing. Ambiental Ing. Agronómica Enfermería Economía Derecho Contaduría Publica Cine y Audiovisuales Biología Antropología Administración de Empresas

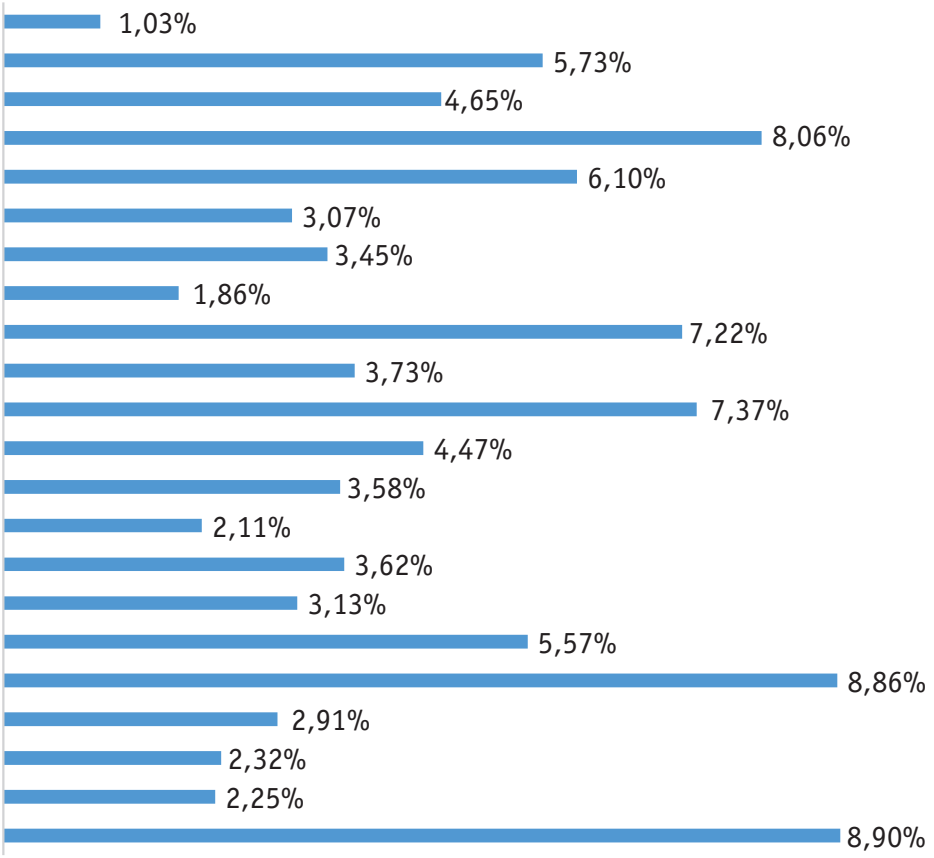

0,00\%1, 00\% 2,00\%3, 00\% 4,00\%5, 00\% $6,00 \% 7,00 \% \quad 8,00 \% 9,00 \% 10,00 \%$

Fuente: autores con base cifras de Marenco, O. (2013)

También se pueden establecer consideraciones sobre la proporción de cada grupo social beneficiado por la política de acción afirmativa de la Universidad. Desde esta perspectiva, se encontró que los bachilleres desplazados y los afrocolombianos son las comunidades que reciben la mayor parte de los beneficios por exoneraciones, cerca del $60 \%$ de las exoneraciones otorgadas a los grupos estudiantiles vulnerables entre el 2005 y 2011-I, tuvo como beneficiarios a la población desplazada y afrocolombiana según el Figura 5.
Entre tanto, las madres cabeza de hogar y los bachilleres provenientes de familias con bajos ingresos económicos (monitorias académica y administrativa) participan en el grupo de beneficiarios con una proporción que oscila entre el $10 \%$ y el $12 \%$. Mientras que sólo un $9 \%$ de la población vulnerable exonerada, hace parte de las comunidades indígenas, es decir equivalente tan solo a 22 personas pertenecientes a este tipo de comunidad de un total de 396 beneficiadas entre el 2005 - 2011. 
Políticas de acceso de la población vulnerable a la educación superior, una visión desde la experiencia de la Universidad del Magdalena

\section{- Figura 4.}

Valor de exoneraciones en matrícula académica. Cifras en miles de millones de pesos. Período 2005 - 2011

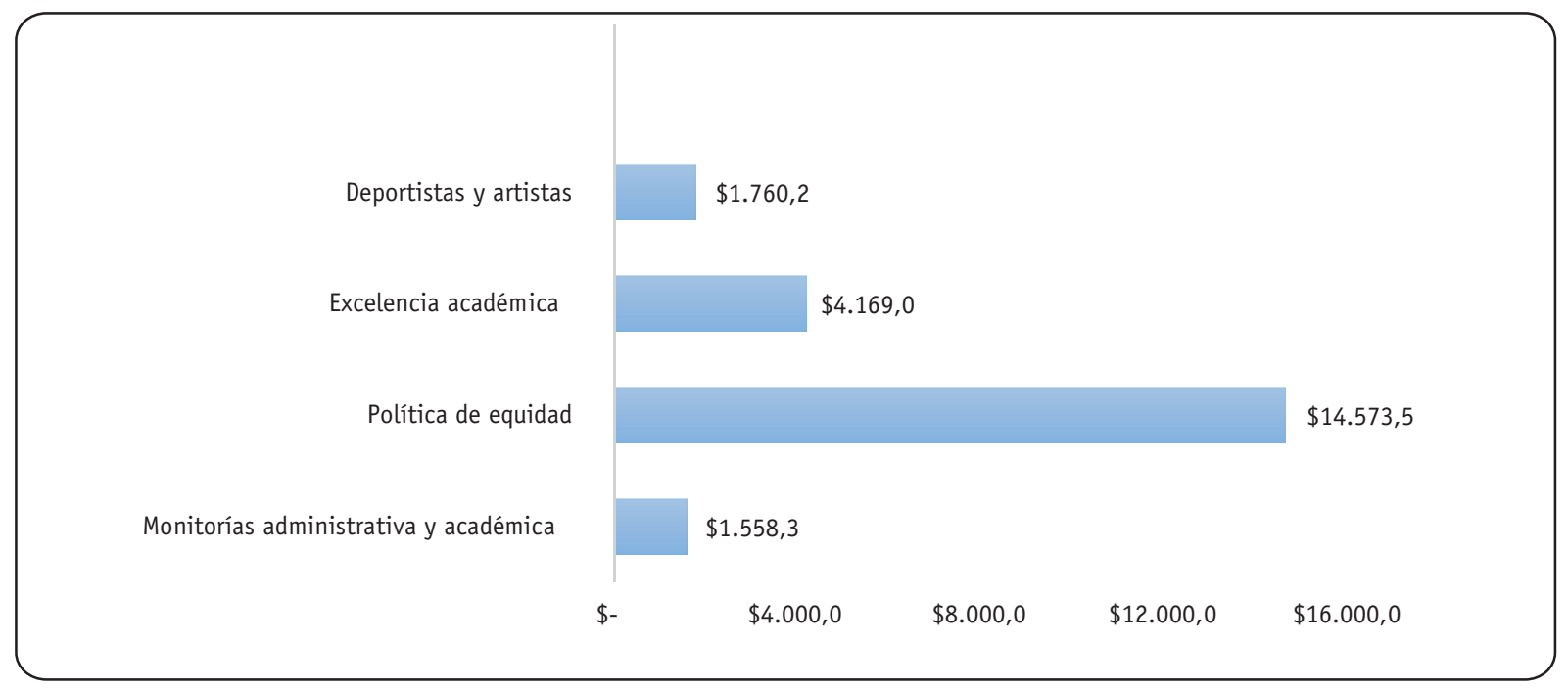

Fuente: Figura de los autores con base en información del Grupo de Admisiones, Registro y Control Académico de la Universidad del Magdalena $2005-2011$

\section{Figura 5.}

\section{Porcentaje de estudiantes exonerados en la Universidad del Magdalena, según comunidad a la que pertenecen $7.2005-2011-\mathrm{I}$}

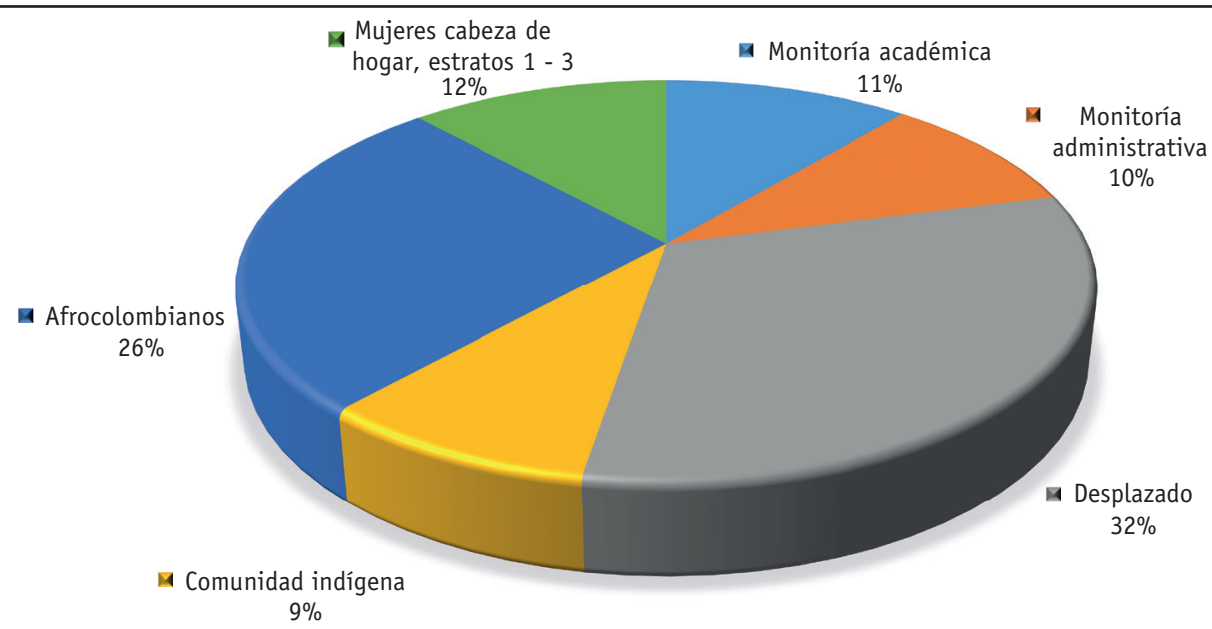

Fuente: Figura de los autores con base en información del Grupo de Admisiones, Registro y Control Académico de la Universidad del Magdalena $2005-2011$

7 Se incluyen en esta figura las categorías monitorias académicas y administrativas por cuanto hacen parte del conjunto de acciones afirmativas dirigidas a la población vulnerable que, en este caso, beneficia a estudiantes de bajos recursos económicos. 


\section{Principales características socioeconómicas y académicas de la población vulnerable en la Universidad del Magdalena}

Con el estudio titulado "Análisis de los programas de equidad en el acceso y la permanencia de los estudiantes de población vulnerable ${ }^{8 ",}$ la Universidad del Magdalena desarrolló una descripción de los aspectos socioeconómicos y académicos representativos de las comunidades vulnerables. Con base en dicho trabajo, en esta sección se presentan los datos más sobresalientes que permiten conocer las condiciones que caracterizan a esta parte de los estudiantes. Se puede afirmar que la mayoría de los estudiantes pertenecientes a las comunidades en desventaja, ingresan a la Universidad bajo condiciones socioeconómicas muy susceptibles.

En primer lugar, en la tabla 3 se puede observar que más del $80 \%$ de los estudiantes proviene de escuelas oficiales, lo que representa un alto riesgo para la permanencia de estos grupos en la Universidad, ya que los resultados de la política pública educativa en el departamento del Magdalena no generan gran impacto en el desarrollo de competencias y conocimientos en los estudiantes.

Tal consideración se puede corroborar con las calificaciones ICFES obtenidas por la población en estudio. En promedio, cerca del $90 \%$ de los universitarios principiantes, se encuentran en la categoría de calificación media y baja. Tan solo poco más del $10 \%$, ha obtenido un puntaje ICFES alto. Estas cifras generan indicios de que, posiblemente, esta parte de la población estudiantil enfrentará problemas en el rendimiento académico derivados de una preparación de baja calidad.

-Tabla 3.

\section{Principales características socioeconómicas y académicas de la población vulnerable en la Universidad del Magdalena}

\begin{tabular}{|l|c|c|}
\hline \multirow{2}{*}{ Género } & Masculino & $49 \%$ \\
\hline \multirow{2}{*}{ Tipo de colegio } & Femenino & $51 \%$ \\
\hline & Público & $82 \%$ \\
\hline \multirow{2}{*}{ Estrato } & Privado & $18 \%$ \\
\hline & 1 & $34 \%$ \\
\hline & 2 & $40 \%$ \\
\hline ICFES & 3 & $23 \%$ \\
\hline
\end{tabular}

Fuente: Tabla de los autores con base en información del Grupo de Admisiones, Registro y Control Académico de la Universidad del Magdalena 2005 - 2011.

8. Título completo: Análisis de los programas de equidad en el acceso y la permanencia de los estudiantes de población vulnerable en los programas de pregrado de la Universidad del Magdalena durante los años comprendidos entre 2005 - 2011-I 
Políticas de acceso de la población vulnerable a la educación superior, una visión desde la experiencia de la Universidad del Magdalena

En todo caso, tanto el puntaje ICFES obtenido por los incipientes universitarios como el tipo de escuela de procedencia, no se pueden analizar de manera aislada. Hay que mencionar que, además, las condiciones económicas no son muy favorables.

De la figura 6 se puede inferir que, entre el 2005 y 2011-I, el 72\% de la población en estudio proviene de familias cuyos ingresos mensuales no superaban los $\$ 600,000$ pesos MLV (Moneda legal vigente). De tal modo, se puede decir que hay una predisposición desfavorable que limita, por razones económicas, a esta población para recibir una educación secundaria de mejor calidad; obsérvese que, en el Tabla 3, las proporciones de estudiantes egresados de colegios privados presenta una relativa equivalencia con los bachilleres que obtuvieron un puntaje alto en las pruebas ICFES.

\section{- Figura 5.}

Distribución porcentual del ingreso económico familiar de estudiantes clasificados como población vulnerable. Período 2005 - 2011

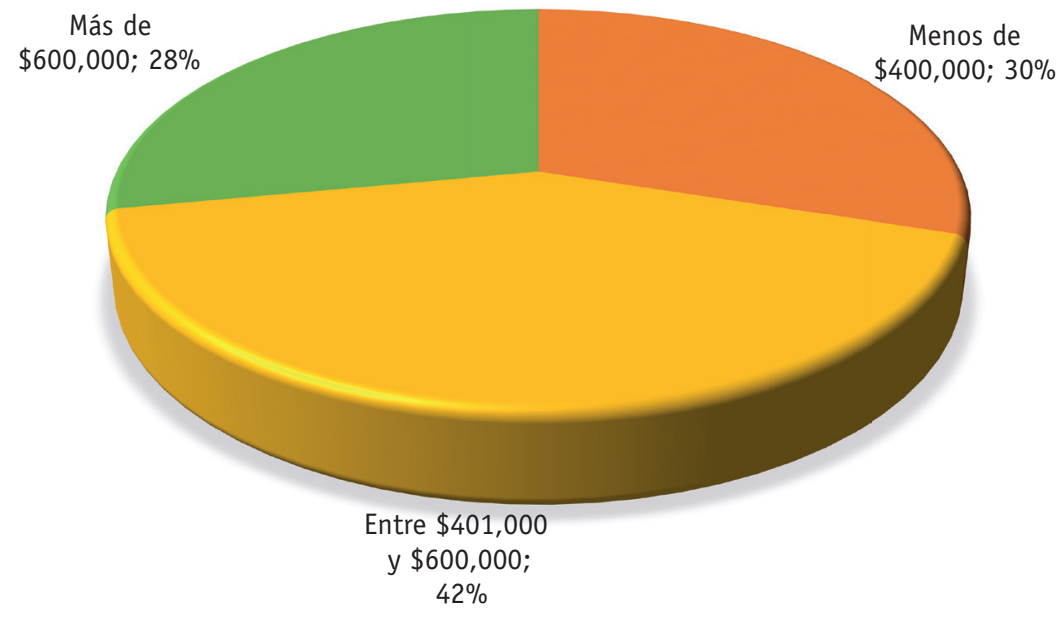

Fuente: figura de los autores con base en información del Grupo de Admisiones, Registro y Control Académico de la Universidad del Magdalena $2005-2011$.

\section{Conclusión y Discusión}

Del estudio se concluye que las acciones afirmativas constituyen el principal mecanismo de las IES para facilitar el acceso de la población históricamente marginada. Las probabilidades de permanencia de los estudiantes incrementan $8 \%$ por cada política de acción afirmativa ejecutada.

Entre las principales políticas de inclusión de la población vulnerable se encuentran las admisiones o cuotas mínimas especiales, las becas y subsidios 
focalizados, el acompañamiento académico, el seguimiento a los egresados, los cursos preuniversitarios y la eliminación de estándares mínimos de acceso.

En la Universidad del Magdalena el $66 \%$ de los recursos disponibles; equivalente a $14.573,5$ mil millones de pesos, para la ejecución de las políticas de acción afirmativa, se destinan al financiamiento de la política de equidad, es decir, son recursos girados directamente al beneficio de la población vulnerable de la Universidad.

Otra conclusión importante es que existe una alta concentración de la política de exoneraciones en la Universidad, el $61 \%$ de éstas se entregan en las facultades de Ingeniería y Ciencias Empresariales, aunque se debe tener en cuenta que estas unidades académicas reciben la mayoría de estudiantes matriculados.

Además, acerca de las características socioeconómicas y académicas se puede resumir que la mayoría de los estudiantes de la Universidad del Magdalena provienen principalmente de colegios públicos $(80 \%)$, con una calificación ICFES en su mayoría media y baja (90\%).

Finalmente, existe una gran concientización por parte de las autoridades académicas de la Universidad del Magdalena acerca de la importancia de la ejecución de políticas que nivelen los estándares de equidad entre los estudiantes, sólo entre el 2005 y el 2011 la Universidad gastó alrededor de $\$ 22,000$ millones de pesos para disminuir las dificultades a las que se enfrenta la población vulnerable.

\section{Referencias Bibliográficas}

Gómez, V. \& Celis, J. (2009). Crédito educativo, acciones afirmativas y equidad social en la educación superior en Colombia. Revista de Estudios Sociales. № 33. Pp. $106-$ 117. Agosto de 2009, Bogotá.

Marenco, 0. (2013). Caracterización de la población estudiantil beneficiada de las políticas de apoyo para el acceso y permanencia en la Universidad del Magdalena período 2005 - 2011-I. Tesis no publicada. Pp. 86. Universidad del Magdalena.

Mercado, A. (2013). Análisis de los programas de equidad en el acceso y la permanencia de los estudiantes de población vulnerable en los programas de pregrado de la Universidad del Magdalena durante los años comprendidos entre 2005 - 2011-I. Universidad del Magdalena. Santa Marta.

Ministerio de Educación Nacional, MEN (2013). Cómo rinde la educación superior: así vamos en acceso y permanencia de la educación superior. Junio de 2013.

MEN, (2005). Lineamientos de política para la atención educativa a poblaciones vulnerables. Dirección de Poblaciones y Proyectos Intersectoriales. Bogotá. Julio, 2005.

Organización para la Cooperación y el Desarrollo Económicos, OCDE (2012). La educación superior en Colombia 2012. Evaluaciones de políticas nacionales de educación.

Reiter, B. y Lezama, P. (2013). Transferencias condicionales y políticas de acción afirmativa en Latinoamérica: la diferencia que políticas de inclusión pueden hacer. Revista Investigación \& Desarrollo. Vol. 21. No 2. Artículos de Reflexión. Universidad del Sur de La Florida.

Sánchez, F., Quirós, M., Reverón, C., y Rodríguez, A. (2002). Equidad social en el acceso y permanencia en la universidad pública determinantes y factores asociados. Documento CEDE, 2002-16. Universidad de Los Andes. Bogotá D.C. 